\title{
Las mujeres en las academias de ciencias del mundo
}

\author{
Lillian Alvarez \\ lilliam@ceniai.inf.cu \\ Academia de Ciencia de Cuba \\ Havana, Cuba.
}

\section{RESUMEN}

El presente texto discute las categorías desarrolladas por el feminismo y los estudios de género para explicar la condición de las mujeres y los coloca en tensión respecto de las realidades de las mujeres afrodescendientes e indígenas de América Latina. Con ello se pretende mostrar las fronteras de las categorías con la finalidad de avanzar en los estudios a partir de contextos históricos específicos que permitan otorgar nuevas conexiones entre la teoría y las realidades de las mujeres latinoamericanas. Se presenta información actualizada sobre todo un movimiento que está ocurriendo en el mundo, con datos, estadísticas, conceptos, informes recientes de varios organismos internacionales y reflexiones sobre la participación de las mujeres en las ciencias. Se compilan informes, argumentos, hechos, que se ilustran con resultados recientes, cuantitativos y cualitativos, de encuestas realizadas en mas de 70 Academias de ciencias del mundo, como expresión de uno de los espacios de "élite" donde son elegidos los especialistas de mas alto nivel en cada país, poniendo en evidencia la baja representatividad de las mujeres en las academias y que aunque ha habido avances, aun no son suficientes. Se destaca en particular el caso de la Academia de Ciencias de Cuba como una de las de más altos porcentajes de mujeres tanto en su membresía como en su consejo directivo y las razones para este hecho.

PALABRAS CLAVE: Mujer en la ciência. Mujer en la academia. Inclusión de género en la ciencia. 


\section{INTRODUCCIÓN}

El artículo recoge una investigación realizada sobre el estado del arte acerca de la participación de las mujeres en la ciencia a partir de la aportación e involucración directa de la autora por mas de 20 años en programas, comités, redes y encuestas regionales y mundiales.

La mas reciente actualización se hizo a propósito de un taller organizado por la Consejo internacional para las Ciencias, ICSU, de conjunto con el Comité de ética y responsabilidad de la ciencia, celebrado en México en Abril, 2016 con el objetivo de llamar la atención y redactar una nota asesora para ICSU, sobre el tema de género en la investigación científica con foco en la movilidad de las mujeres y la internacionalización de la ciencia. En el programa del taller se vieron distintas aristas y enfoques, desde la ciencia vlsta y hecha por mujeres desde Europa hasta un panorama de la participación de las mujeres en la ciencia del mundo en desarrollo, presentado este último tema por la autora.

Resulta difícil colocar en una publicación la riqueza de los resultados, análisis y recomendaciones de varios informes elaborados desde comisiones e instituciones del mas alto nivel en el mundo, por lo que el trabajo se limita a subrayar, informar, ilustrar un problema común: en pleno avance del siglo 21 , aun las mujeres no participan plenamente en los logros de la ciencia que se denomina como universal.

Se resalta el caso particular de Cuba y su Academia de ciencias como ejemplo de inclusión de las mujeres en su membresía como resultado de una voluntad política de casi 6 décadas de Revolución socialista.

\section{Problema}

¿Tenemos realmente un problema? Lamentablemente la respuesta es si.

Veamos algunas cifras y hechos:

- Las 3/5 partes de las personas mas pobres del mundo son mujeres y niñas

- El 70\% de las 130 millones de personas que no estudian en el mundo, son féminas

- Las 2/3 partes de los 960 millones de analfabetos en el mundo, son mujeres.

\section{Sexo, poder, influencia científica}

Los siguientes gráficos del 2015, muestran el promedio global de presencia mujeres en los Parlamentos de todo el mundo, representadas por un $22 \%$ y por otro lado cuántas mujeres ocupan carteras ministeriales, con un porciento mucho menor para un $17 \%$. 
Figura 1. Mujeres y hombres en los Parlamentos del mundo

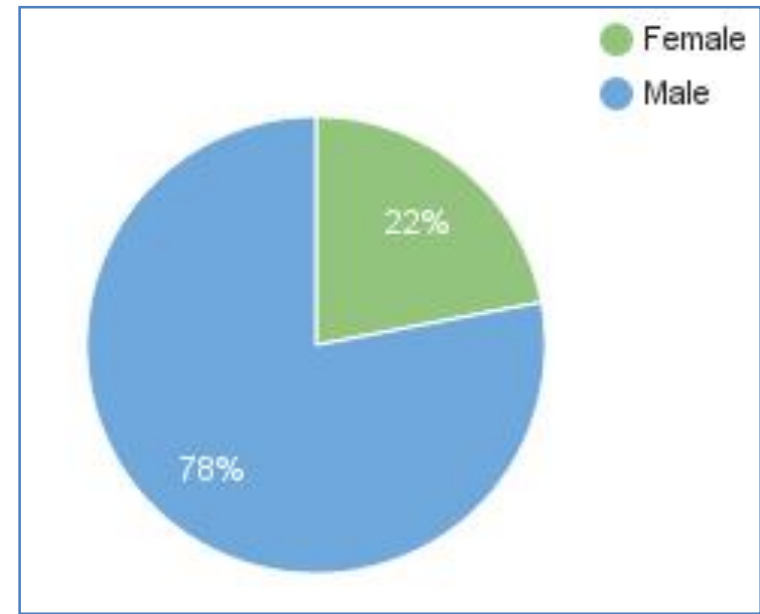

Fuente: UN Women, http://www.unwomen.org/en/what-we-do/leadershipand-political-participation/facts-and-figures

Figura 2. Mujeres y hombres que son ministros del mundo

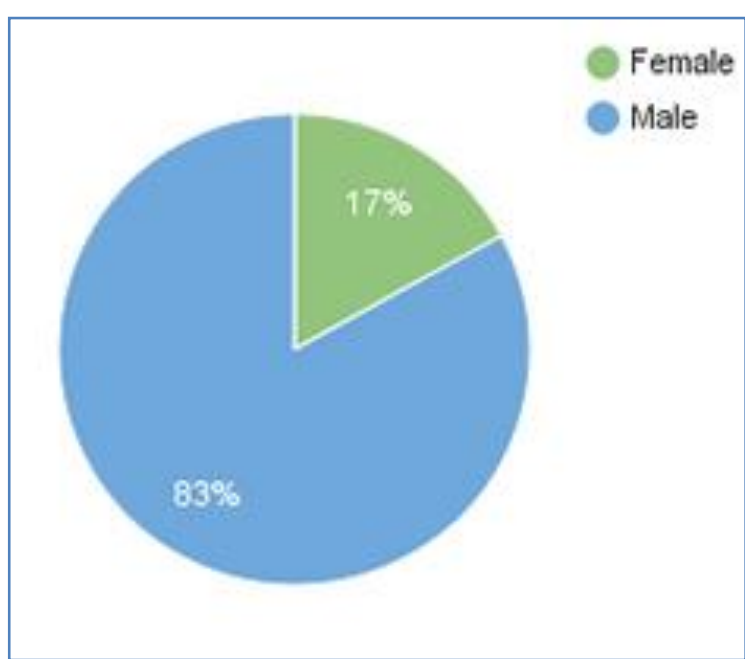

Fuente: UN Women, http://www.unwomen.org/en/what-we-do/leadershipand-political-participation/facts-and-figures

Entonces ¿tenemos un problema? Sí, somos las mas pobres y las menos empoderadas, siendo la mitad de Humanidad.

-Una meta de las Naciones Unidas para el Desarrollo sostenible

De las 17 metas establecidas por las Naciones Unidas para el desarrollo sostenible (17 metas para transformar el mundo) la número 5 expresa: 
Meta 5:

Alcanzar la equidad género y empoderar a las mujeres y a las niñas

En el informe del PNUD, Powerful Synergies, se expresa que el acceso paritario y control de los recursos no solo mejora la vidas de los individuos, de las familias y de las naciones sino que ayuda a garantizar la sostenibilidad del entorno. Lo interesante de esta publicación es que se presentan una colección de evidencias que exploran la interconexión que existe entre la equidad de género y el desarrollo sostenible a través de diversos sectores, desde la educación, la salud, la seguridad alimentaria, los cambios climáticos, la cultura energética, los derechos humanos, los hábitos y patrones de consumo. No es posible hablar de desarrollo sostenible sino potenciamos la equidad de género.

Pero lo más valioso es compartir que este informe contiene recomendaciones para las políticas y para los que deben ejecutarlas y practicarlas que aseguren integrar la equidad de género y la plena participación de las mujeres.

Figura 3 - powerful synergies

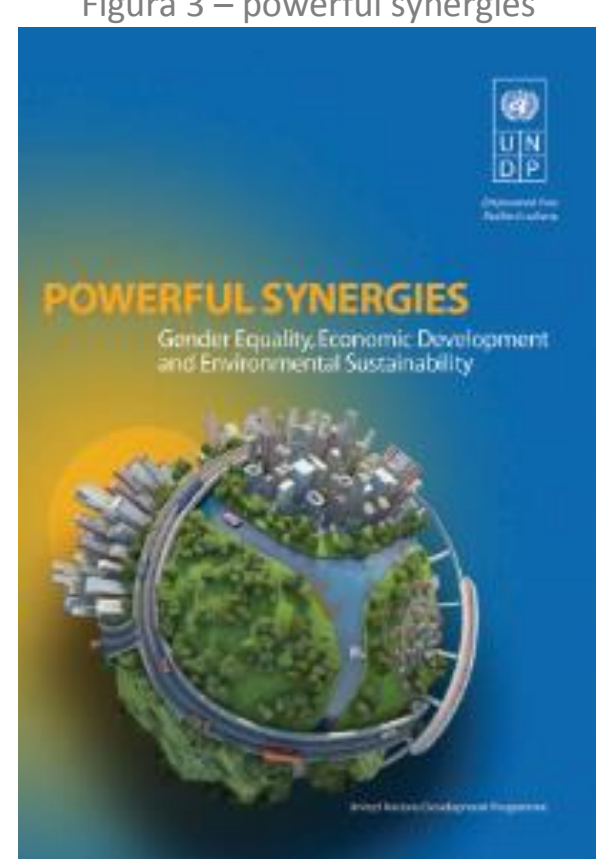

Fuente:http://www.undp.org/content/undp/en/home/librarypage/womensempowerment/powerful-synergies/

En particular para las mujeres de ciencia la ONU acaba de establecer en el 2016, el 11 de Febrero de cada año como el Día Internacional de las Mujeres y las Jóvenes en las Ciencias, explicitando que las investigaciones siguen mostrando que las mujeres continúan siendo excluidas de las ciencias y se constata en el número de graduadas de ciencias en el mundo que es mucho menor que el de los hombres. Por supuesto que estos indicadores por países se diferencian pero el panorama global, -incluyendo a los millones de jóvenes de los países pobres, eufemísticamente llamados "del mundo en desarrollo" reflejan, avanzando ya la segunda década del siglo, estas terribles realidades de inequidad. 


\section{DESARROLLO}

¿Qué dice la UNESCO en su último informe sobre la ciencia?

En el Informe del a UNESCO sobre la Ciencia Hacia el 2030 se registra una frase lapidaria "The other half of human capital still a minority"- La otra mitad del capital humano, aun es minoría.

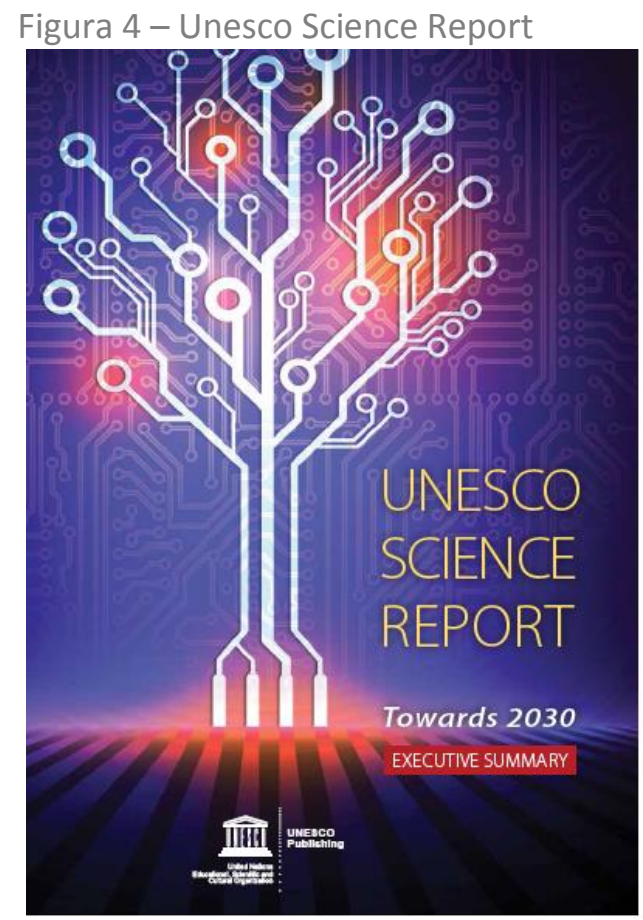

Fuente: Informe de la UNESCO

http://unesdoc.unesco.org/images/0023/002354/235407s.pdf

El informe nos explica que cuando un país enfrenta el dilema de contar con una masa crítica de científicos necesaria para enfrentar los problemas del desarrollo económico y social, inmediatamente sus actitudes en cuanto a la incorporación de las mujeres cambia. En eso Cuba es un ejemplo desde 1959 y eso lo subraya la autora. Pero además la UNESCO nos revela que algunos estados árabes tienen más mujeres que hombres estudiando ciencias naturales, ciencias médicas y ciencias agrícolas en las universidades y que el $37 \%$ de los investigadores en el mundo árabe son mujeres, más que en la Unión Europea donde son el 33 \%.

El informe de la UNESCO expresa que las regiones del mundo que tienen mas mujeres en la investigación, son el Sur-este europeo con un $49 \%$ (interesante observar que son en su mayoría los países ex socialistas, subrayo yo), los estados caribeños, Asia y América latina con un $44 \%$ mientras que son solo el $14 \%$ en Japón y el $18 \%$ en Corea del Sur.

En general las mujeres siguen siendo una minoría en el mundo de la investigación, tienen menos acceso a los fondos, y se desarrollan con mucha mayor dificultad en su crecimiento profesional y acceso a grados y categorías científicas superiores, reflejo del efecto tijera que es común en todos los países, incluida Cuba. Véase la clásica tijera, en la Figura 5, del CSIC español, en rojo las mujeres, 
en azul los hombres, se gradúan en similares porcentajes y luego se separan al ascender ellos a categorías o plazas docentes y científicas superiores y ellas descienden y por supuesto entonces son peor remuneradas. Lo mismo sucede con el Sistema nacional de investigadores en México. Según aumenta el nivel (y los estímulos) aparecen menos mujeres.

Figura 5. Las investigadoras en España, datos del CSIC, 2014

Personal Investigador CSIC 2014

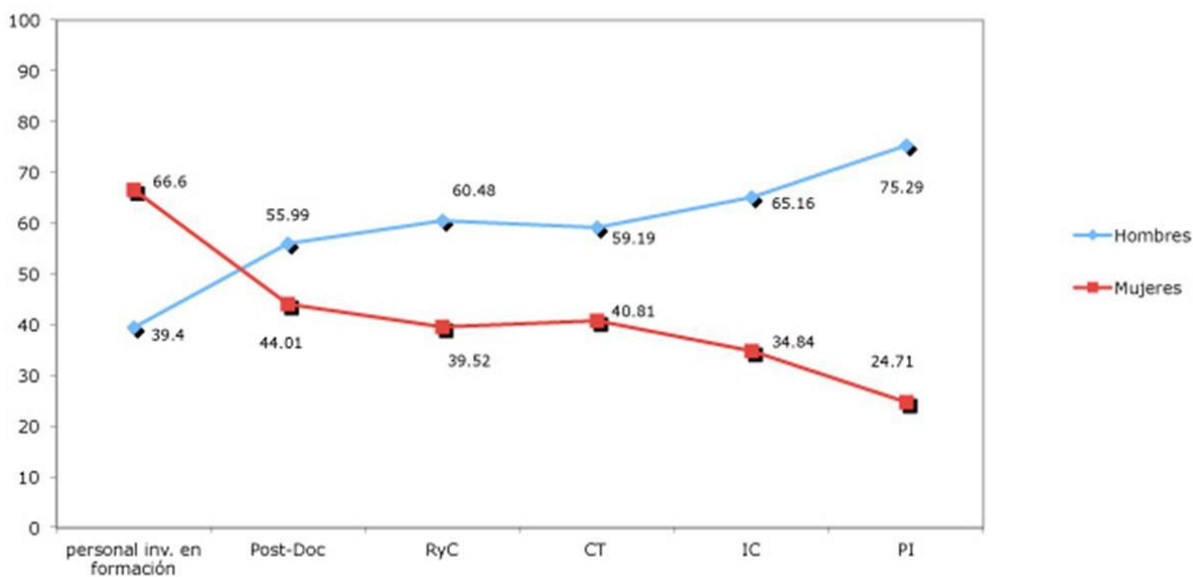

Fuente: http://cdnb.20m.es/sites/113/2016/02/Tijera CSIC 2014.jpg

Un artículo reciente de autores españoles revela como titular "Una mujer por cada nueve hombres en la élite de la ciencia europea" denunciando que las mujeres acceden en mucha menor cuantía a los fondos millonarios de los programas científicos mas prestigiosos de la Unión Europea y una de las explicaciones es que "muchas mujeres dejan de competir por los fondos porque tienen otras prioridades", ¿`será cierto o hay sesgo o discriminación en esa frase?

\section{RESULTADOS DE LAS ENCUESTAS}

\section{Encuestas en las membresías de las Academias de ciencias}

Las estadísticas no lo dicen todo, eso es sabido, pero una tabla o un gráfico bien hecho dice más que cien discursos. Además, persisten percepciones subjetivas de que las mujeres no tenemos dificultades y que hemos alcanzado altos estándares en le ciencia y en la tecnología. Ya observamos que en América somos mas o menos la mitad del total de los investigadores, pero, ¿cómo se comporta el acceso a las Academias de Ciencias? Aunque las diferentes Academia de las Américas tienen diferentes procederes para la elección de sus miembros, si es cierto que en sus membresías están las élites científicas, los mas relevantes, los mas eminentes, los que mas han aportado.

Entonces, desde la Red Interamericana de Academia de ciencias a través del programa de Mujeres en las ciencias- del cual la autora ha sido co-directora hasta 2016- se planteó realizar una encuesta e investigar cómo se comporta la presencia de las mujeres en las Academias de la región, desde Canadá, EEUU, México hasta Brasil, Argentina, incluyendo la Academia de ciencias del Caribe. 
En la Survey of women in the academies of the Americas se puede consultar completo el cuestionario realizado a todas academias de las Américas y los interesantes resultados y análisis realizados.

Figura 6 - Survey of Womwn in the Academies of the Americas

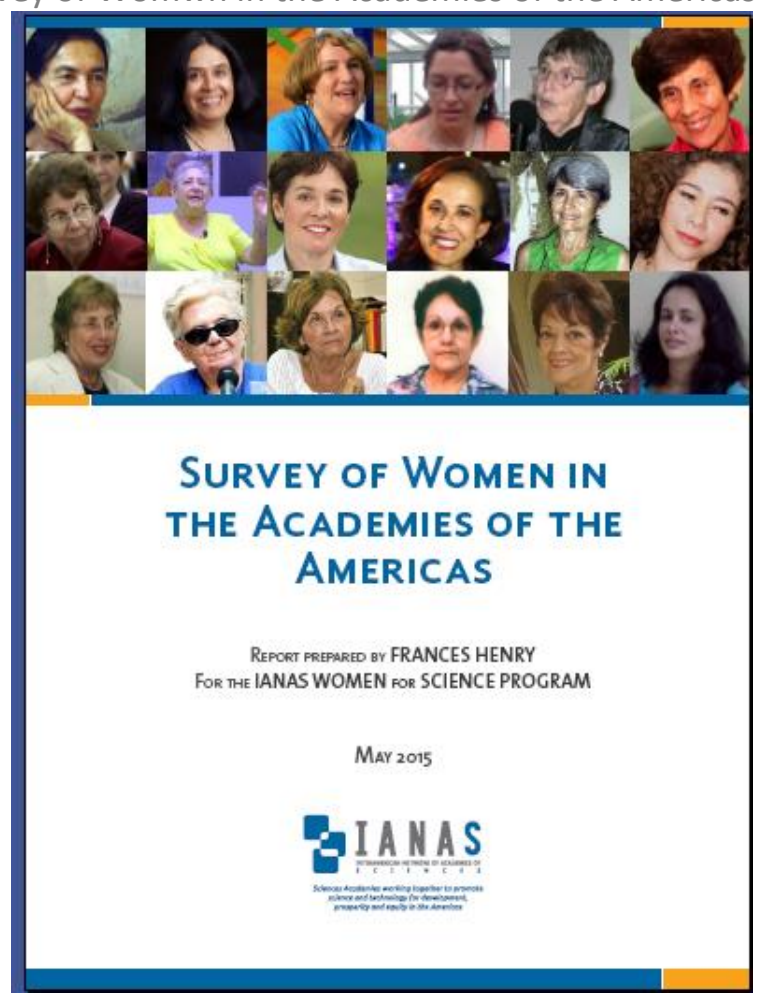

Fuente: IANAS Survey

http://www.ianas.org/books/books_2015/women/survey-of-women.pdf

Antes de mostrar algunos de los resultados de la encuesta comparto algunas expresiones al ver el libro, de lo que las expertas llaman "sexismo benevolente": 1) Felicidades Lilliam, veo muchas caras conocidas en la portada, 2) ¿Cómo te las arreglaste para que el diseñador te colocara en el centro de la portada?, 3) ¿Por qué te dedicas a estos temas en lugar de seguir investigando e incrementando tu currículo como matemática? .... 


\section{En Estados Unidos}

El gráfico muestra que la presencia de mujeres en la Academia nacional de ciencia ha ido creciendo, pero aun en el 2014 no sobrepasaba el $14 \%$.

Figura 7. Porciento de mueres por años en la Academia Nacional de Ciencias de EEUU.

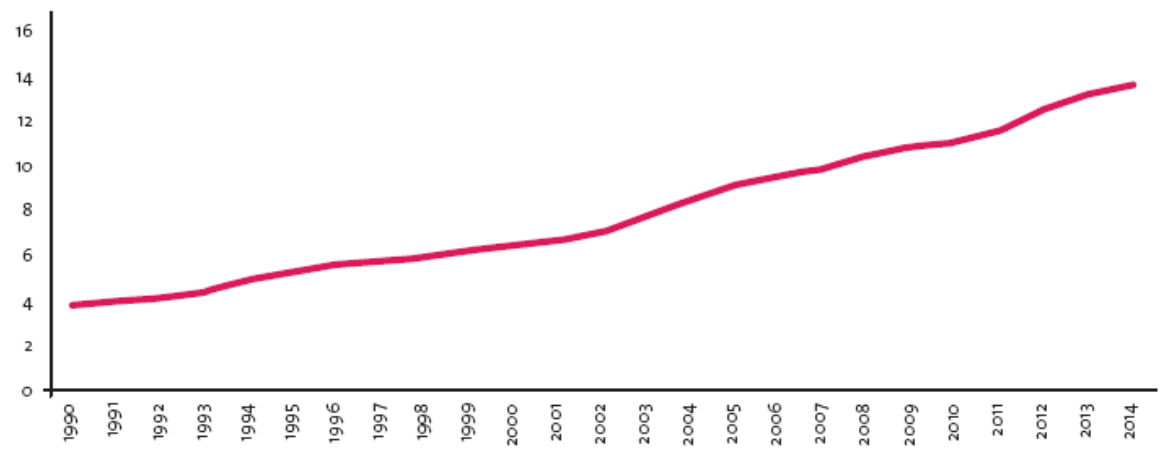

Fuente: IANAS Survey

http://www.ianas.org/books/books_2015/women/survey-of-women.pdf

Proporción de mujeres por ramas de la ciencia en todas la Academias de las Américas

La siguiente figura muestra que ellas aparecen en mayoría en las ciencias sociales, en Biología, en Ciencias médicas y de la salud y como es sabido son minoría en la Física y en la Matemática. En Química y Astronomía están en igual proporción.

Figura 8. Proporción de hombres y mujeres por ramas de las ciencia en las Academias de ciencias de las Américas.

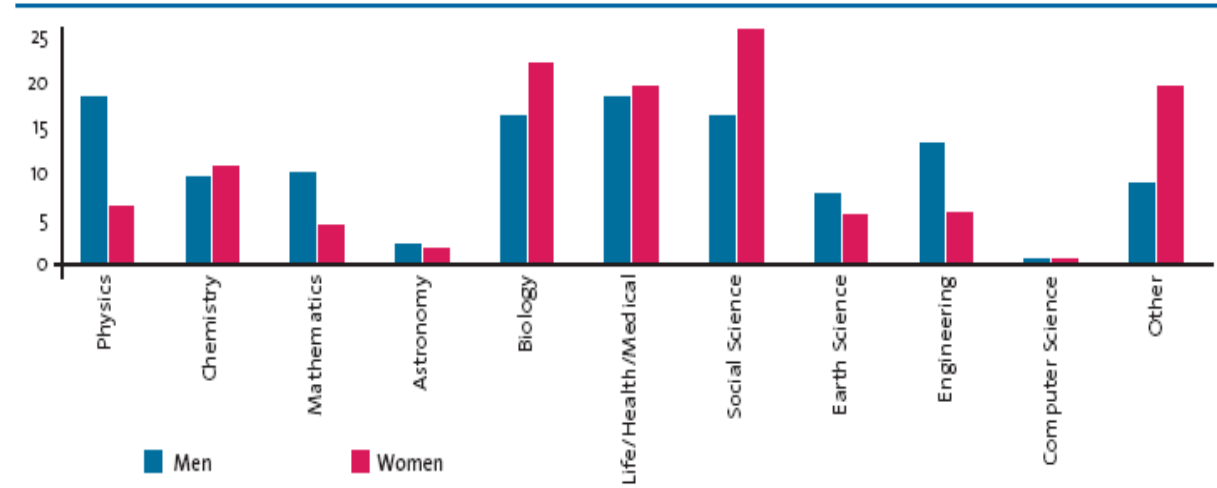

Fuente: IANAS Survey

http://www.ianas.org/books/books_2015/women/survey-of-women.pdf

\section{Mujeres en las juntas directivas de las Academias}

Se observan en la Figura 9 barras verdes muy altas, esto es, muchas mujeres en los secretariados de las academias- eso si, "somos buenas secretarias", pero aparecemos mucho menos en los consejos de gobierno o juntas directivas-en los puestos de poder-, barras rojas. Se revela la Academia de Ciencias de Cuba con mejor equilibrio y presencia de mujeres en junta directiva y en el secretariado. El 
caso de Panamá no es comparable porque lo que existe es la APANAC La Asociación para el avance de la ciencia, cuya membresía no es electiva, es abierta.

Figura 9. Porciento de mujeres por países: miembros de las juntas de gobierno (consejos directivos) y su presencia en los secretariado de las Academias.

Women members (\%)

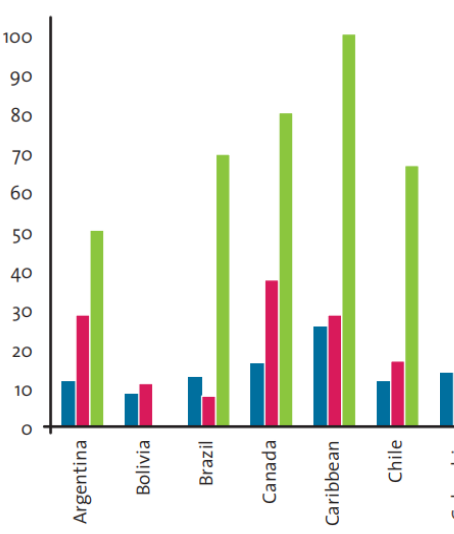

Women on Governing Council (\%)

Women in Secretariat (\%)

Fuente: IANAS Survey

http://www.ianas.org/books/books_2015/women/survey-of-women.pdf

La encuesta de IANAS no sólo reflejó lo que dicen las estadísticas con indicadores cuantitativos, varios de ellos conocidos desde una percepción subjetiva pero no probados numéricamente; sino que también elaboró preguntas cualitativas a las Academias que, con sólo formularlas contribuyen a profundizar la conciencia y cultura de género y sobre la necesidad de incluir a las mujeres en las membresías y en los puestos de poder de las Academias.

Algunas preguntas fueron:

¿Está su Academia consciente de la baja participación de las mujeres en la ciencia y qué acciones se están tomando?

- ¿Su Academia promueve activamente los asuntos de género en sus estructuras, en los puestos de decisión y en programas y proyectos?

- ¿ ¿Qué acciones serían las mas eficientes para promover que las jóvenes entren en carreras de ciencia? ¿Promueve este asunto en las escuelas?

- ¿ ¿Su Academia tiene una política de género?

Esa pregunta sobre si la Academia tiene o no una política explícita de género arrojó que no siempre tener un documento oficial escrito resuelve el problema, porque por ejemplo la Academia cubana no lo tiene y es la número uno en la Región. Sólo el $22 \%$ de las Academias dijeron contar con una política de género y otro caso es Chile que la tiene, pero solo tiene un $12 \%$ de mujeres en su membresía, aunque es justo decir que Chile y Venezuela acaban de elegir a una mujer como Presidenta de la Academia de Ciencias, y Cuba la tuvo por 9 años, la Dra. Rosa Elena Simeón, (1985-1994).

Una académica cubana Guadalupe Guzmán fue entrevistada y aparece en el libro de IANAS "Mujeres científicas en las Américas: sus historias inspiradoras", mostrando y subrayando los logros de las mujeres científicas en Cuba. 
Además, el Programa de IANAS decidió realizar también un libro sobre Jóvenes científicas Un futuro brillante para las Américas, con el subtítulo "descubre por qué y cómo estas jóvenes mujeres decidieron ser científicas, tú puedes ser una de ellas" y donde una Joven asociada de la ACC fue entrevistada, Noslen Hernández, experta en Química-Matemática. De su entrevista los editores decidieron subrayar una oración: Si yo pudiera transmitirle algo a las jóvenes que decidan ser científicas sería una combinación balanceada de tres aspectos: motivación, dedicación y ética, Ref. [7].

Ha sido la autora de este trabajo el punto focal del Programa de mujeres en la ciencia de la Red IANAS de 2010 a 2016 y preside actualmente el grupo de Cuba de la Red Iberoamericana de ciencia, tecnología y género del programa CYTED.

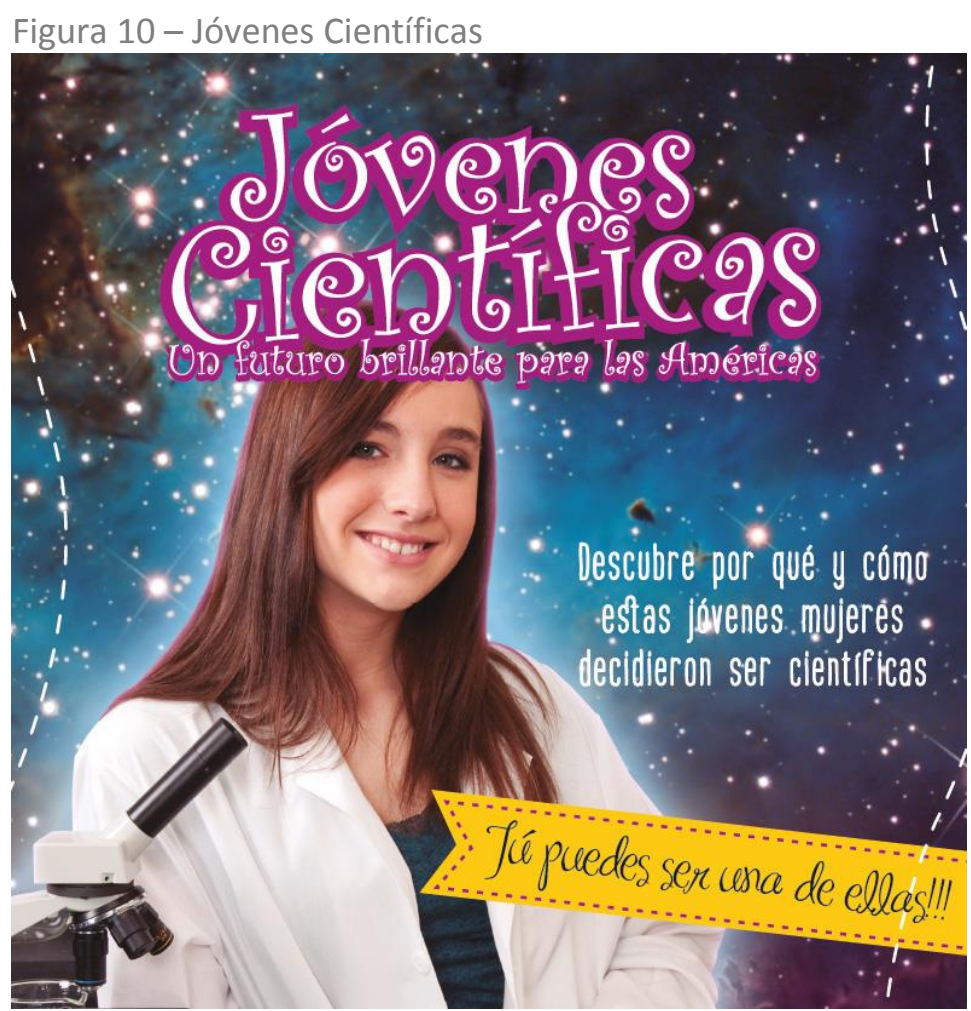

Fuente:Todos los libros de IANAS pueden ser bajados del sitio: http://www.ianas.org/index.php/books

La encuesta mundial de IAP

En el año 2015, la Red global de academias de ciencias -partenariado interacademia, IAP (anteriormente Inter Academy Panel) publicó por primera vez una encuesta para estudiar la representación de las mujeres en sus membresías, en sus juntas de gobierno y en las actividades generales de las academias. En el reporte publicado se expresa que los hallazgos o resultados y las recomendaciones deberían usarse y diseminarse, darse a conocer a los tomadores de decisiones para desarrollar estrategias y establecer políticas para incrementar esa participación de las mujeres. La Red global IAP está compuesta por 111 academias del mundo y respondieron las encuesta 69 academias de ciencias nacionales y tres de carácter regional como IANAS y la Academia de ciencias del Caribe. 
El texto mismo de la encuesta fue el fruto de una amplia consulta y un esfuerzo colectivo donde la autora tuvo una importante participación así como en el aporte de las cifras y respuestas cualitativas de la Academia de Ciencias de Cuba cuyo texto definitivo de la encuesta fue aprobado en su Consejo directivo.

Figura 11- Women for sciences

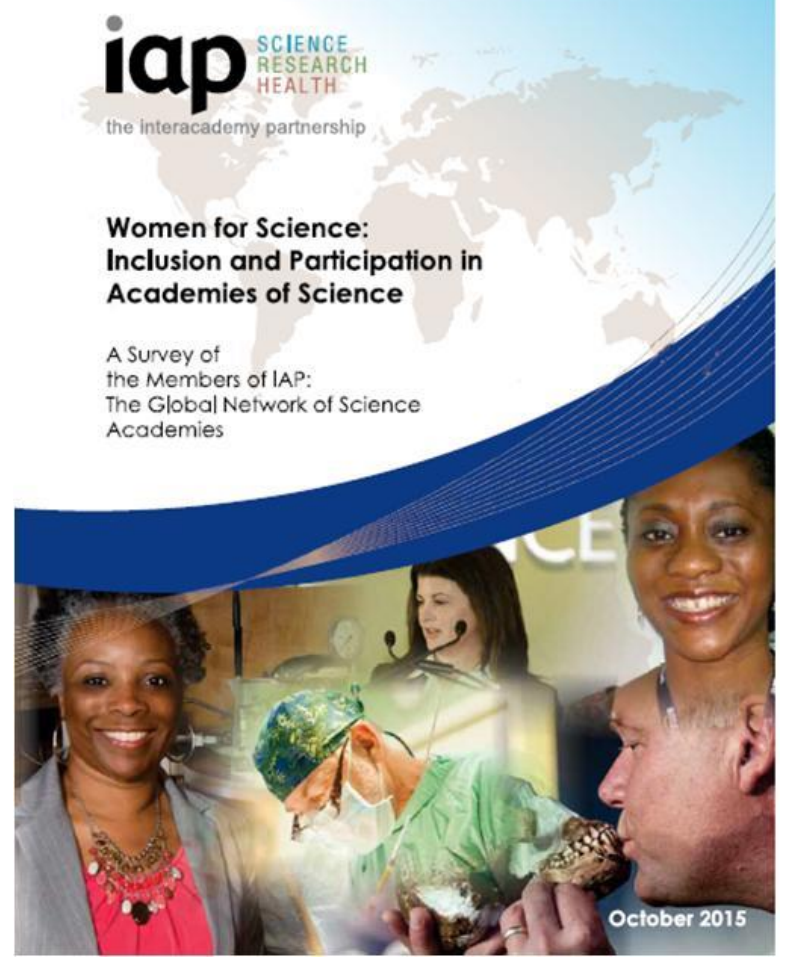

Fuente: http://www.interacademies.net/File.aspx?id=29834

Figura 12. Porcentaje de mujeres en todas academias encuestadas según las características de sus membresías

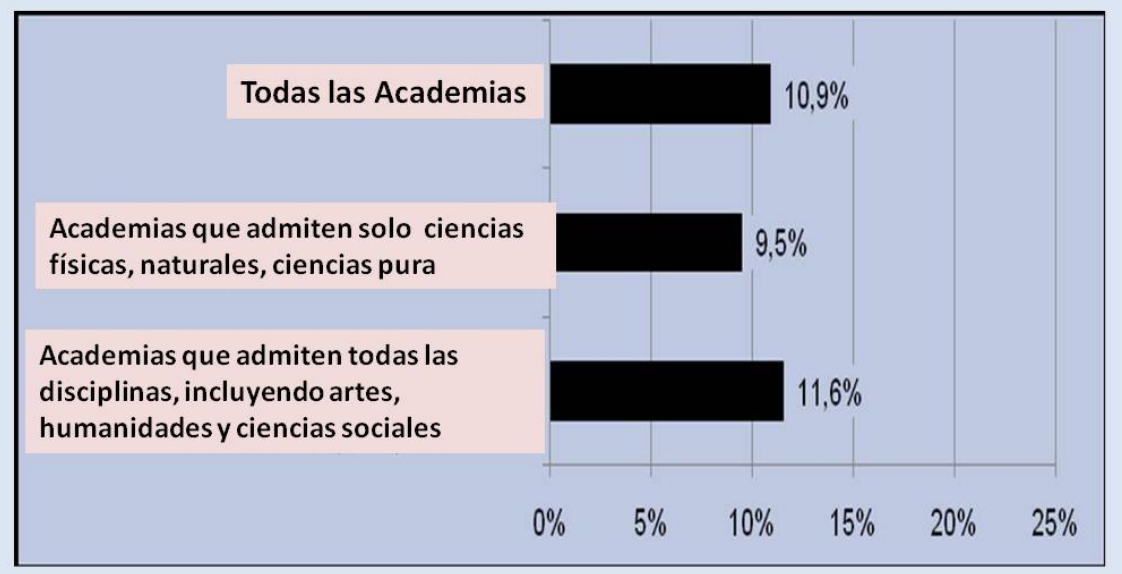

Fuente: http://www.interacademies.net/File.aspx?id=29834 
Se observa en la Figura 12 que el promedio global de mujeres en las academias es muy bajo, de $10,9 \%$, y si se trata de academias que solo están compuestas por las ciencias físicas, naturales y ciencias básicas, es mucho menor, 9,5\%. Aun en aquellas academias que incluyen las ciencias sociales, las humanidades y las artes, el promedio es $11,6 \%$, con lo que se revela la falta de equidad en la elección de los miembros y con ello la falta de reconocimiento y visibilidad de eminentes mujeres de ciencias que no son promovidas, o recomendadas o elegidas para las academias nacionales.

Interesante es subrayar que el propio reporte del IAP en su resumen ejecutivo valida que las dos academias que tienen mayores porcentajes de mujeres entre sus miembros son la de Cuba, con un $27 \%$ y la Academia del Caribe que tiene un $26 \%$. También otras academias de las Américas tienen cifras de mujeres que superan el $10 \%$, pero siempre revelando que ellas están mejor representadas, y lo mismo sucede en Cuba, en las ciencias sociales y menos en las ingenierías y en las matemáticas donde son solo el 5\%, y en Cuba menos también en la Biomedicina.

Que sean menos las mujeres científicas que llegan a las academias desde las ingenierías y las matemáticas es hasta cierto punto lógico porque ellas son minorías en el mundo en esas profesiones.

\section{La Academia de Ciencias de Cuba}

Es innegable que el Socialismo como sistema abrió las puertas de la inclusión y la justicia social, primero en los países del este de Europa y luego en China, Vietnam, Cuba. En particular para la ciencia, las mujeres fueron incluidas en las carreras y en las instituciones científicas con una voluntad política y sistemas de apoyo para su plena incorporación, con la creación de círculos infantiles, escuelas con seminternados, posibilidades de formación de pre y posgrado en instituciones de excelencia en el extranjero, entre otros. Aún hoy los países ex socialistas y las repúblicas ex soviéticas mantienen, como un hecho ya incluido en su cultura, alto porcientos de mujeres en los sectores de la ciencia y la tecnología que superan los de la Europa occidental.

La Revolución cubana desde 1959 tuvo esa vocación y estrategia desde sus mismos inicios, siendo ellas las que mas contribuyeron a erradicar el analfabetismo y las que mas acudieron a formarse como maestras y poblar las universidades y los centros científicos, dándose un fenómeno de feminización de las universidades cubanas en las década de los 1970 y los 80.

La Academia cubana tuvo una mujer como Presidenta de 1985 a 1994, la Dra. Rosa Elena Simeón y desde 1999, a partir de la Conferencia Mundial sobre la Ciencia para el Siglo XXI, auspiciada por la UNESCO y el Consejo Internacional para la Ciencia celebrada en Budapest, se tomó el acuerdo de crear la Comisión de mujeres académicas como comisión permanente que vela y monitorea la presencia de las mujeres, contribuye a reconocer sus resultados, promueve a la incorporación de las mas jóvenes hacia las carreras de ciencia.

Esta comisión también coordina el Capítulo nacional de la Organización mundial de mujeres de ciencia del mundo en desarrollo, OWSD, otorga cada dos años los premios Sofia Kovalievskaia a mujeres relevantes en las ciencias básicas, promueve concursos para las mas jóvenes, representa a la Academia en redes regionales para los temas de las mujeres en las ciencias, divulga en los medios a 
las mas relevantes, haciéndolas visibles como modelos de rol a seguir, ejecuta tareas de investigación para compilar sus historias de vida, realiza acciones conjuntas con instituciones, cátedras y con la Federación de Mujeres cubanas, FMC. Esta última organización contó con el liderazgo de Vilma Espín y ha contribuido a concretar mediante legislaciones, políticas, estrategias, la incorporación de las mujeres cubanas en todos los sectores de la vida económica y social. Es también la FMC la que vela y monitorea anualmente el cumplimiento de los Acuerdos de la Cumbre de Beijing en todos los organismos e instituciones a todos los niveles.

Un reciente estudio sobre la presencia de las mujeres en los premios anuales de la academia ha reflejado que ellas siempre están en una proporción menor que los hombres, o sea hay menos mujeres como líderes de las investigaciones que resultan premiadas y por ende primeras autoras. Cuando a la cifra se le suman las mujeres que son co-autoras o parte de los otros autores, entonces aumentan los números, mostrando que ellas están presentes en casi todos los equipos de excelencia de Cuba y que son acreedores de los premios máximos que se otorgan a la ciencia nacional.

Por otro lado hay que referirse a que todos los años el comportamiento del número de premios por cada una de las 5 secciones de la Academia, son mayores en Biomedicina y en Ciencias naturales y exactas, y los premiados en general de las ciencias agrarias, sociales y técnicas son menos. Con ello se expresa entonces que al ser las dos últimas ciencias las que menos premios reciben tienen menos oportunidad las mujeres de estar representadas en ellos. Por ello no es posible decir que en general las mujeres cubanas están menos presentes en las ciencias sociales, sino al contrario, en Cuba ellas son una gran mayoría en estas ciencias, como sucede en casi todo el mundo, pero al escudriñar solo en las que llegan a ser premiadas, están en menor proporción. Algo similar sucede con las ciencias técnicas-mujeres ingenieras y arquitectas- que en Cuba en general están en proporción equitativa, pero mucho menos representadas en los premios de la Academia.

Otra arista interesante es que si afinamos el análisis y observamos "con el microscopio" sólo la Sección de ciencias naturales y exactas, vemos ahí a muchas biólogas, químicas, bioquímicas, geógrafas y con mucha menor presencia las mujeres físicas y matemáticas, lo cual también es común en otras instituciones académicas y países en general Ref. [9].

No obstante todos los avances y logros que han tenido las mujeres científicas cubanas, aun subsisten los problemas de una sociedad patriarcal organizada en torno al poder simbólico y real de los hombres. Aun se reflejan los problemas de techo de cristal, piso pegajoso, efecto pipeta, efecto tijera, sexismo hostil violencia de género, sexismo benevolente, todos conceptos definidos por las expertas en género y referenciados por Alvarez (2012, 2001, 2002, 2015), Pérez-Sedeño (2001), Fernadez-Rius (2007) y Lara Junco (2015). 


\section{CONSIDERACIONES FINALES}

Dijo Hypatia de Alejandría "Conserva celosamente el derecho a reflexionar, porque incluso el hecho de pensar erróneamente, es mejor que no pensar en absoluto". Marie Curie sentenció: la Humanidad necesita de soñadores y el gran Sócrates también dijo: sólo si se sabe, se puede divisar el bien".

Sirvan estas aseveraciones para concluir esta contribución y subrayar que las mujeres no somos minorías, que somos la mitad de la Humanidad, y las madres y abuelas de la otra mitad y que la ciencia no es completa si en ella no participamos las mujeres. 


\title{
Mulheres nas academias de ciências do mundo
}

\author{
RESUMO \\ Se apresenta informações atualizadas sobre o movimento que está \\ acontecendo no mundo, com fatos, estatísticas, conceitos, relatórios recentes de \\ diversas organizações internacionais e reflexões sobre a participação das mulheres \\ nas ciências. Compila-se informes, argumentos, fatos que são ilustrados por \\ resultados recentes de cunho qualitativo e quantitativo em pesquisa realizada em \\ mais de 70 academias de ciências do mundo, como uma expressão de um dos \\ espaços de "elite", onde eles são eleitos os especialistas do mais alto nível de cada \\ país, com destaque para a baixa representação das mulheres nas academias e \\ embora tenha havido progressos, ainda não são suficientes.Destacou-se em \\ particular o caso da Academia de Ciências de Cuba como uma das que apresenta \\ os maiores percentuais de mulheres, tanto em sua composição e seu conselho de \\ administração bem como as razões para isso. \\ PALAVRAS-CHAVE: Mulher na ciência; Mulher na academia; Inclusão de gênero na \\ ciência.
}

\section{Women in the science academies in the world}

\begin{abstract}
Updated information is presented on the participation of women in sciences in the world showing reflections, analysis, data and statistics. Facts, arguments, qualitative and quantitative are compiled in particular from two important surveys published recently about the presence of women in more than 70 academies of sciences in the world, having into account that the academies are representatives of those spaces were most prominent scientists are elected en each country. The crucial results is the extremely low percentage of women in the academies showing that there have been some advancements but not sufficiently. The Cuban Academy of Sciences is highlighted as the first in the world in the percentage of women in the membership and in the governing council and the facts for that successful.
\end{abstract}

KEYWORDS: Women in sciences, women in the academies, gender inclusion in sciences. 


\section{AGRADECIMENTOS}

La autora reconoce el trabajo de la Comisión de mujeres de la Academia de ciencias de Cuba, el aporte del Programa de mujeres en la ciencia de IANAS y de la Red CYTED Red Iberoamericana de Género, Ciencia y Tecnología (RIGCYT). Agradece a Lindamir Salete y a Carla Cabral, por su interes en la publicación em Los Cuadernos de esta investigación.

\section{REFERÊNCIAS}

ALVAREZ, Lilliam, Cuando Las Mujeres suman mejor. Revista Ciencia, Innovación y Desarrollo, Vol. 6, No.3, 2001.

ALVAREZ, Lilliam, M. Valero, M. del C. Battle. La mujer en los Premios de la Academia de Ciencias de Cuba, (2000-2014): presencias, ausencias, presente y futuro, no prelo.

ALVAREZ, Lilliam. ¿Más poder y competitividad para su empresa? La fórmula es incluir a las mujeres. Revista Nueva Empresa, GECYT, La Habana, 2015.

ALVAREZ, Lilliam. Ser mujer científica ¿o morir en el intento?, Editora ACADEMIA, La Habana, Cuba, 2012.

ALVAREZ, Lilliam. Women doing hard sciences in the Caribbean. Proceedings of the IUPAP Conference on Women in Physics. UNESCO, Paris, October, 2002, AEP Conference Proceedings Volume 628, pp. 151-152., Eds. Beverly K. Hartline and Dongqi Li.

CSIC. Disponível em:

http://cdnb.20m.es/sites/113/2016/02/Tijera_CSIC_2014.jpg. Acesso em: 10/fev./2017.

FERNÁNDEZ RIUS, Lourdes, Género y mujeres académicas: ¿Hasta dónde la equidad?, Facultad de Psicología, Universidad de La Habana, 2007.

IANAS. IANAS Books. Disponível em: http://www.ianas.org/index.php/books. Acesso em: 10/fev./2017.

IANAS. Mujeres Científicas en las Américas: sus historias inspiradoras. Disponível em:

http://www.ianas.org/books/MUJERES_CIENTIFICAS_EN_LAS_AMERICAS_opt.pdf Acesso em : 10/fev./2017.

IANAS. Survey of Women in the Academies of the Americas. Disponível em: http://www.ianas.org/books/books_2015/women/survey-of-women.pdf. Acesso em: 15/jan./2017.

IANAS. Women for Science Program. http://www.ianas.org/index.php/books. 
IAP. Women for Science: Inclusion and participation in Academies of Scences, Academy of Science of South Africa (ASSAf), PUBLISHER Pretoria. Disponível em: http://www.interacademies.net/File.aspx?id=29834. Acesso em: 15/jan./2017.

LARA JUNCO, Teresa, ¿Cuánto y cómo trabajan las mujeres en Cuba?, 22 de mayo, 2015. Disponível em: http://oncubamagazine.com/author/teresa_lara/. Acesso em: 10/dez./20176.

PÉREZ SEDEÑO, Eulalia. Las mujeres en el Sistema de Ciencia y Tecnología. Estudios de casos. OEI, España, 2001.

UNESCO. Informe de la UNESCO sobre la ciencia. Disponível em: http://unesdoc.unesco.org/images/0023/002354/235407s.pdf. Acesso em: 15/jan./2017.

UNITED NATIONS DEVELOPMENT PROGRAMME. Powerful Synergies: Gender Equality, Economic Development and Environmental Sustainability. Disponível em: http://www.undp.org/content/undp/en/home/librarypage/womensempowerment/powerful-synergies/. Acesso em: 10/fev./2017.

UNWOMEN. Facts and figures: Leadership and political participation. Disponível em:http://www.unwomen.org/en/what-we-do/leadership-and-politicalparticipation/facts-and-figures. Acesso em: 20/fev./2017.

Recebido: 03 abr. 2017

Aprovado: 08 nov. 2017.

Como citar:

ALVAREZ, Lillian. Las Mujeres en las Academias de Ciencias del Mundo. Cad. Gên. Tecnol., Curitiba, v. 10, n. 36, p. 28-44, jul./dez. 2017.

Correspondência:

Lillian Alvarez. Academia de Ciencia de Cuba. Calle Cuba, no 460 entre Amargura y Tte Rey, La Habana Vieja, La Habana, Cuba.

Direito autoral:

Este artigo está licenciado sob os termos da Licença Creative Commons-Atribuição 4.0 Internacional. 